\title{
Article \\ Standardized Biomechanical Investigation of Posture and Gait in Pisa Syndrome Disease
}

\author{
Elisa Panero ${ }^{1,2, *(\mathbb{D})}$, Ugo Dimanico ${ }^{2}$, Carlo Alberto Artusi ${ }^{3}\left(\mathbb{D}\right.$ and Laura Gastaldi ${ }^{4}(\mathbb{D}$ \\ 1 Department of Mechanical and Aerospace Engineering, Politecnico di Torino, 10129 Turin, Italy \\ 2 Department of Surgical Sciences, Università degli Studi di Torino, 10124 Turin, Italy; ugo.dimanico@unito.it \\ 3 Department of Neuroscience, Università degli Studi di Torino, 10124 Turin, Italy; carloalberto.artusi@unito.it \\ 4 Department of Mathematical Sciences, Politecnico di Torino, 10129 Turin, Italy; laura.gastaldi@polito.it \\ * Correspondence: elisa.panero@polito.it
}

check for updates

Citation: Panero, E.; Dimanico, U.; Artusi, C.A.; Gastaldi, L. Standardized Biomechanical Investigation of Posture and Gait in Pisa Syndrome Disease. Symmetry 2021, 13, 2237. https://doi.org/ $10.3390 /$ sym 13122237

Academic Editor: Paul Sung

Received: 27 September 2021

Accepted: 18 November 2021

Published: 23 November 2021

Publisher's Note: MDPI stays neutral with regard to jurisdictional claims in published maps and institutional affiliations.

Copyright: (c) 2021 by the authors. Licensee MDPI, Basel, Switzerland. This article is an open access article distributed under the terms and conditions of the Creative Commons Attribution (CC BY) license (https:/ / creativecommons.org/licenses/by/ $4.0 /)$.

\begin{abstract}
Pisa syndrome is one of the possible postural deformities associated with Parkinson's disease and it is clinically defined as a sustained lateral bending of the trunk. Some previous studies proposed clinical and biomechanical investigation to understand the pathophysiological mechanisms that occur, mainly focusing on EMG patterns and clinics. The current research deals with the assessment of a standardized biomechanical analysis to investigate the Pisa syndrome postural effects. Eight patients participated in the experimental test. Both static posture and gait trials were performed. An optoelectronic system and two force plates were used for data acquisition, while a custom multi-segments kinematic model of the human spine was used to evaluate the 3D angles. All subjects showed an important flexion of the trunk superior segment with respect to the inferior one, with a strong variability among patients (range values between $4.3^{\circ}$ and $41.0^{\circ}$ ). Kinematics, ground reaction forces and spatio-temporal parameters are influenced by the asymmetrical trunk posture. Moreover, different proprioception, compensation and abilities of correction were depicted among subjects. Considering the forces exchanged by the feet with the floor during standing, results highlighted a significant asymmetry $(p$-value $=0.02)$ between the omo and contralateral side in a normal static posture, with greater load distribution on the same side of lateral deviation. When asked to self-correct the posture, all patients demonstrated a reduction of asymmetry, but without stressing any statistical significance. All these aspects might be crucial for the definition of a PS patients' classification and for the assessment of the efficacy of treatments and rehabilitation.
\end{abstract}

Keywords: standing posture; gait analysis; multi-segment spine model; 3D kinematics; spatiotemporal parameters; Parkinson's disease; Pisa syndrome

\section{Introduction}

Parkinson's disease (PD) is a chronic and developing neurodegenerative pathology characterized by several motor and non-motor symptoms. PD is associated with a progressive neuronal loss of the substantia nigra and other brain structures [1]. Individuals affected by PD exhibit notable symptoms, often asymmetric, such as gait impairment, unbalance, and tremors during different motion activities [2].

Previous observational and clinical studies pointed out that a third of patients with PD had a postural deformity at lower limbs, trunk and neck. Severe abnormities of posture and spinal alignment might lead to important disability and difficulty in conducting daily activities. These spinal deformities were classified and defined based on their main characteristics and the most common are camptocormia, antecollis, scoliosis and Pisa syndrome [3,4]. Pisa syndrome (PS) is clinically defined as a sustained lateral bending of the trunk of $10-15^{\circ}[5,6]$. The real prevalence of PS in Parkinson's disease is still unknown due to the unclear diagnosis. Previous literature reported a prevalence from $2 \%$ to $90 \%$, but a recent experimental analysis made in a multicenter Italian study has estimated a prevalence of $8.8 \%$ [7]. This postural alteration is often mismatched with scoliosis due 
to the similarity on plane and body segment involved in the deformity. Nevertheless, the two are clinically different, both for the mechanisms and possible therapies [4]. The combination of lateral trunk deviation and vertebral rotation in PS is reversible, therefore a physiological lateral flexion can be achieved by a passive mobilization or supine posture; while in scoliosis it is fixed $[4,8,9]$.

Biomechanical procedures and motion capture instrumentation such as optoelectronic and inertial sensor systems have been largely adopted in clinical fields in an attempt to quantify objective parameters that cannot only be evaluated with observational and clinical analysis [10-12]. Gait and posture analysis has been widely used in different pathologies [13-15]. Experimental assessment of human motion revealed a fundamental role in studying pathologies, testing the effects of treatments and rehabilitation, supporting the clinical specialists in understanding and decision-making processes $[16,17]$. Focusing on Parkinson's disease, gait analysis and movement dysfunctions have been deeply analyzed in the past, pointing out gait asymmetries, risk of falling, reduced walking speed, alteration of joint kinematics and important impact of cognitive loads on performing movements [18,19].

Some previous literature studies proposed clinical and biomechanical investigation in order to understand the pathophysiological mechanisms that occur in Pisa syndrome [20-22]. Among them, in 2013, Tinazzi and colleagues proposed an experimental study for the description of electrophysiological and imaging of PS, in which patients underwent EMG of paraspinal lumbar and thoracic muscles, X-ray and MRI of the trunk. Based on the results, two main patterns had been identified and associated with PS. The first one was characterized by hyperactivity of lumbar paraspinals ipsilateral to the trunk leaning side, while the second pattern presented hyperactivity of lumbar paraspinals contralateral to the trunk leaning side. Moreover, in the first pattern, patients presented ipsilateral or contralateral hyperactivity of thoracic paraspinals, while, in the second pattern, all patients showed hyperactivity of contralateral thoracic paraspinals [23]. In 2015, Geroin and colleagues investigated balance in PD and PS patients, monitoring the center of pressure (CoP) displacement in a standing posture with opened and closed eyes [24]. Patients' results were compared with the healthy control group. A significant difference was pointed out between PS and control group due to the increased displacement of CoP in mediolateral and anteroposterior directions. Moreover, shifting of $\mathrm{CoP}$ was contralateral to the direction of trunk bending in five patients, ipsilateral in the others; the different behavior was probably due to the different postural strategies adopted. The study highlighted the difficulties in achieving good postural alignment in PS patients and suggested a rehabilitation program as a possible solution to improve postural control [24]. Another important study on the assessment of posture in PS patients dealt with the use of a wall goniometer as a screening tool to detect and classify the postural misalignments in Parkinson's disease [25]. Tinazzi and colleagues demonstrated that the wall goniometer is a quick and low-cost instrument for the quantification of misalignment and validated that method on 283 PD patients with different postural abnormalities. Despite the high level of agreement with the selected gold standard, some underestimations were obtained, especially in lower camptocormia [25].

Although numerous studies assessed muscles activation [26-29] and postural misalignment $[30,31]$ in PD patients, only a few of them also investigated gait. Tramonti and colleagues investigated gait both in Pisa syndrome and camptocormia by measuring spatio-temporal parameters and angular kinematics in the sagittal plane. Results were compared with a control group. Correlation between spatio-temporal parameters and clinical scales pointed out that stride and step length were associated with reduced functional abilities. Kinematic data in the sagittal plane revealed a reduced range of motion in all lower extremities' joints [32].

Despite the innovative and biomechanical approach, some limitations might be stressed in the evaluation of gait only in the sagittal plane, as in the separation of right and left sides instead of omolateral and contralateral sides with respect to trunk deviation. Moreover, the evaluation of the spine kinematics and the bodyweight distribution on two 
feet might underline the fundamental consequences of Pisa Syndrome on human motion. Several multi-segments models of the human spine and algorithms have already been developed [33-36], however, none have been used for the investigation of Pisa Syndrome. Moreover, the lack of a standardized procedure for the analysis and the absence of quantification of objective biomechanical parameters results might lead to discrepancies among the different studies.

The current research deals with the definition and validation of a standardized biomechanical methodology to investigate the Pisa Syndrome postural effects and consequences during standing posture and gait. A customized multi-segments model of the human spine developed and validated in a previous study [37] was adopted for the assessment of spinal kinematics. Static and dynamic tasks were performed by PS patients. Biomechanical variables as spatiotemporal parameters, 3D spine and lower limbs kinematics and ground reaction forces were quantified and considered as parameters of interest.

\section{Materials and Methods}

\subsection{Subjects}

Ten patients affected by Parkinson's Disease (PD) and Pisa Syndrome (PS) were recruited for the study, but two of them were excluded during the experimental tests due to the perceived difficulty in performing motion tests proposed. All participants met the following inclusion criteria: diagnosed idiopathic Parkinson's disease, diagnosed Pisa syndrome, absence of orthopedic comorbidity and dementia, ability to walk without any type of support. Eight subjects (five male and three female) participated in the experiment after giving their informed consent. The study was approved by the Local Institutional Review Board. All procedures were conformed to the Helsinki Declaration.

Mean and standard deviation (SD) values of subjects' anthropometric data were evaluated (age: $64.88 \pm 9.64$ years, height: $1.70 \pm 0.12 \mathrm{~m}$, weight: $66.88 \pm 17.66 \mathrm{~kg}$, BMI: $22.9 \pm 3.65 \mathrm{~kg} / \mathrm{m}^{2}$ ). Clinical (age of PD, age of PS) and biomechanical (vertebral level of lateral deviation and side of deviation) characteristics of each PS patient are summarized in Table 1. Based on this data, a preliminary classification of patients can be proposed based on the level of lateral deviation. Indeed, five patients presented the starting point of lateral deviation at lumbar zone (L2-L4), while three patients at the end of thoracic zone (T10-T12).

The kinematic analysis was performed in the so-called "daily-On" therapeutic condition. The antiparkinsonian therapy was maintained stable over the previous month and patients maintained their usual therapeutic scheme. Information on disease stage and dopaminergic therapy of enrolled patients were reported in Table 1.

\subsection{Instruments and Kinematic Models}

Tests were conducted in laboratory setting, in the specialized Movement Disorders Center of "Unità Spinale Unipolare-Città della Salute e della Scienza" in Turin. The total capture volume of the laboratory was $10 \mathrm{~m}$ in length and $5 \mathrm{~m}$ in width.

A motion capture system based on stereophotogrammetry was used for the experimental analysis and data registration. It was composed of eight infrared cameras VICON Bonita $(120 \mathrm{~Hz})$, two video cameras VICON VUE $(50 \mathrm{~Hz})$ and two Kistler force plates $(1000 \mathrm{~Hz})$. A total of 28 passive markers were positioned on human anatomical landmarks in order to define two different human models: Plug-in-Gait lower body model and customized spine model [37].

The customized model [37] is a multi-segment of the trunk, that was previously developed and validated on healthy subjects. However, in the present study, due to the interest in separating the human trunk into two segments in correspondence of the vertebral level where the primary deviation started, the 6-segments kinematic model of human trunk was simplified to 5-segments: pelvis, trunk inferior (inf), trunk superior (sup), neck, and head. A cluster of four markers was used to identify each of the heads (LPHD, RPHD, LAHD, RAHD), trunk superior ( $\mathrm{DEV}_{\mathrm{SUP}}, \mathrm{C7}$, T10, RBACK), trunk inferior 


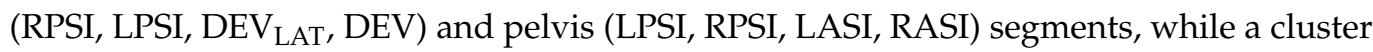
of three markers was used for the neck $\left(C 7, C 5, C_{\text {lat }}\right)$ segment. For each segment, a proper local coordinate system was defined with the $x$-axis oriented in the same direction of gait, $z$-axis in the longitudinal direction, and $y$-axis pointing to the left side of the participant. The convention of YXZ Euler rotations was adopted to define relative angles of each distal segment with respect to its proximal adjacent one. Figure 1 shows the identification of spinal segments (Figure 1A), the positioning and labeling of passive markers (Figure 1B) and the definition of local reference system (Figure 1C). Due to the posterior view of the subject, the anterior markers positioned on the head (LAHD and RAHD) and on the pelvis (LASI and RASI) are not depicted.

Table 1. Clinical and biomechanical characteristics of the Pisa Syndrome patients.

\begin{tabular}{|c|c|c|c|c|c|}
\hline Subjects & $\begin{array}{l}\text { Deviation } \\
\text { Side and } \\
\text { Level }\end{array}$ & $\begin{array}{c}\text { PD } \\
\text { Duration } \\
\text { (Years) }\end{array}$ & $\begin{array}{l}\text { Pisa } \\
\text { Duration } \\
\text { (Years) }\end{array}$ & $\begin{array}{l}\text { Hoenhn } \\
\text { and Yahr } \\
\text { Stage }\end{array}$ & $\begin{array}{c}\text { Dopaminergic } \\
\text { Therapy }\end{array}$ \\
\hline M01 & $\begin{array}{l}\text { Right } \\
\text { L3 }\end{array}$ & 10 & 4 & 2 & $\begin{array}{l}\text { LEDD: } 1344 \\
\text { Levodopa-LEDD: } 1064 \\
\text { DA-LEDD: } 180\end{array}$ \\
\hline F02 & $\begin{array}{l}\text { Right } \\
\text { T12 }\end{array}$ & 11 & 3 & 2 & $\begin{array}{l}\text { LEDD: } 750 \\
\text { Levodopa-LEDD: } 600 \\
\text { DA-LEDD: } 150\end{array}$ \\
\hline F03 & $\begin{array}{l}\text { Right } \\
\text { L3-L4 }\end{array}$ & 27 & 3 & 3 & $\begin{array}{l}\text { LEDD: } 1091 \\
\text { Levodopa-LEDD: } 931 \\
\text { DA-LEDD: } 160\end{array}$ \\
\hline F04 & $\begin{array}{l}\text { Right } \\
\text { T10 }\end{array}$ & 7 & 7 & 3 & $\begin{array}{l}\text { LEDD: } 775 \\
\text { Levodopa-LEDD: } 700 \\
\text { DA-LEDD: } 75\end{array}$ \\
\hline M05 & $\begin{array}{l}\text { Right } \\
\text { L2-L3 }\end{array}$ & 16 & 5 & 2 & $\begin{array}{l}\text { LEDD: } 1675 \\
\text { Levodopa-LEDD: } 1000 \\
\text { DA-LEDD: } 150\end{array}$ \\
\hline M06 & $\begin{array}{l}\text { Left } \\
\text { T12 }\end{array}$ & 6 & 2 & 3 & $\begin{array}{l}\text { LEDD: } 575 \\
\text { Levodopa-LEDD: } 475 \\
\text { DA-LEDD: } 0\end{array}$ \\
\hline M07 & $\begin{array}{l}\text { Right } \\
\text { L2-L3 }\end{array}$ & 3 & 1 & 2 & $\begin{array}{l}\text { LEDD: } 1480 \\
\text { Levodopa-LEDD: } 1330 \\
\text { DA-LEDD: } 150\end{array}$ \\
\hline M08 & $\begin{array}{l}\text { Left } \\
\text { L3-L4 }\end{array}$ & 20 & 20 & 3 & $\begin{array}{l}\text { LEDD: } 1480 \\
\text { Levodopa-LEDD: } 1330 \\
\text { DA-LEDD: } 150\end{array}$ \\
\hline
\end{tabular}
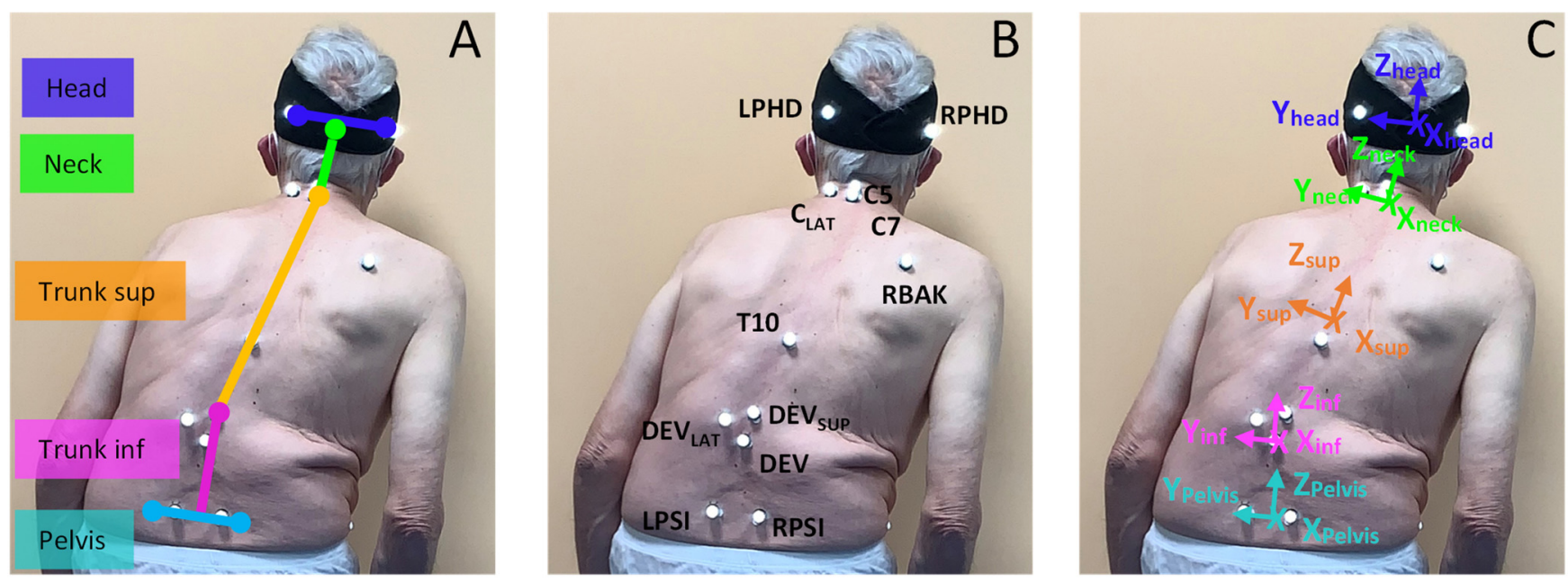

Figure 1. (A) identification of rigid segments of human spine model; (B) positioning and labeling of markers on human spine; (C) local reference system for each rigid segment. 


\subsection{Tasks}

Each subject was first requested to assume a static standing posture for the labeling and scaling of the two models. Then two tasks were performed by patients: static posture and gait trials. During the static posture, the subject was standing with each foot positioned on a separate force plate. Two different trials were recorded: in the first one the subject was asked to assume a natural (relaxed) position, while in the second one he/she was asked to "correct" the lateral bending, by vertically aligning their spine. One trial was performed for each condition and each trial lasted from three to five seconds.

The second task consisted in performing five gait trials. The total length of the path performed by the subject in each trial was $10 \mathrm{~m}$. The participants were asked to walk barefoot back and forth along the path, at a self-selected comfortable speed. Gait events were depicted by means of lateral ankle markers, as proposed by the Plug-in-Gait model.

\subsection{Data Analysis}

Vicon Nexus pipelines were used for the post-processing of markers 3D coordinates. Proper algorithms were used to reconstruct the trajectory and to fill gaps. Plug-in-Gait Dynamic pipeline was used to calculate kinematic data of lower body, while gait events were manually identified based on foot markers trajectories and video recording. A customized Procalc pipeline was developed to estimate the spine kinematics. Customized Matlab ${ }^{\circledR}$ routines were developed to calculate spatio-temporal parameters from gait events, to implement non-parametric statistical data analysis (Wilcoxon test for load distribution and Spearman correlation coefficient for the correlation analysis) and to graphically represent kinematics. Biomechanical results were evaluated separating right and left limbs, but, due to the interest in the effects and asymmetries caused by the PS, the deviation side was considered for the classification of results into omo and contralateral sides with respect to the side of trunk bending.

Static Posture. During the static posture task, both in natural and correct conditions, two objective parameters were considered in the analysis: trunk pose and ground reaction forces. For each subject, the relative angle between trunk superior and trunk inferior segments, and the relative angle between trunk inferior and pelvis segments were estimated in sagittal and frontal planes. Mean values were obtained from all the acquisitions and reported for each subject separately. Ground reaction forces (GRFs) on each foot were acquired and analyzed separately, to consider the different weight distribution on omolateral and contralateral sides. Inter-subjects median and interquartile range values were obtained for each side (omolateral, controlateral) and each condition (natural, correct). Wilcoxon test with level of significance $\alpha=0.05$ was implemented to test the differences between omolateral and contralateral sides, but also between natural and correct conditions ( $p$-value $\leq 0.05)$. The level of correlation between the difference $(\Delta)$ in trunk superior-trunk inferior absolute kinematic angles and the difference $(\Delta)$ in absolute omolateral GRFs due to the correction, was calculated through the Spearman correlation coefficient, both in sagittal and frontal planes. Consistently with the literature, a strong correlation was considered with a coefficient $R \geq 0.7$ [38].

Gait trials. Eleven spatio-temporal parameters (Walking Speed, Stride Time, Step Time, Stance Time, Swing Time, Stride Length, Step Width, Limp Index, Stance Duration, Swing Duration) were calculated. The Limp Index is an adimensional parameter that measures the symmetry of gait and is calculated as the ratio between right and left stance times. Excepted for the Walking Speed $(\mathrm{m} / \mathrm{s})$, all the parameters were assessed separately for the omolateral and controlateral sides and the correspondent absolute difference was estimated for each subject, and then averaged among subjects. Due to the small sample size, the different levels of pathology and the variability in gait performance among subjects, no statistical analysis was implemented for STPs in the current study. Mean and SD values were calculated intra- and inter-subjects. Kinematic analysis of human trunk and lower limbs was assessed separately for each subject, evaluating the mean and SD curves among different gait cycles and different gait trials. Total range of motion (ROM) of lower limbs 
kinematics was estimated along the three axes for each subject, then mean and SD values inter-subjects were valued. The physiological range of motion (ROM) of hip, knee and ankle joints during gait was considered as value of comparison with the attempt to highlight any gait alterations.

\section{Results}

Results are reported and discussed separating the two motion tasks: static posture with natural and correct conditions, and gait.

\subsection{Static Posture}

Table 2 shows the mean relative angles of the trunk segments for each subject separately, both in frontal and sagittal planes during a static standing posture. Figure 2 shows the inter-subjects median and interquartile range values of GRFs on omolateral and contralateral sides, both during natural and correct conditions, highlighting the significant differences estimated with the Wilcoxon test $(\mathrm{z}$-score $=-2.38, p$-value $=0.02$ between omo- and contralateral sides during natural posture). No statistical significance was pointed out for the comparison between omo and contralateral sides during correct posture ( $\mathrm{z}$-score $=-0.98, p$-value $=0.34$ ) and between natural and correct conditions (z-score $=-1.82$ and $p$-value $=0.07)$.

Table 2. Relative spinal angles (proximal-distal) in sagittal and frontal planes during standing.

\begin{tabular}{ccccccccc}
\hline \multirow{8}{*}{ Subjects } & \multicolumn{7}{c}{ Spine Relative Angle $\left(^{\circ}\right)$} \\
\cline { 2 - 8 } & \multicolumn{7}{c}{ Tk sup-Tk Inf } & \multicolumn{2}{c}{ Tk Inf-Pelvis } & Tk Sup-Tk Inf & Tk inf-Pelvis \\
\cline { 2 - 8 } & Natural & Correct & Natural & Correct & Natural & Correct & Natural & Correct \\
\hline M01 & $36.1^{\circ}$ & $14.7^{\circ}$ & $-0.5^{\circ}$ & $6.1^{\circ}$ & $-8.4^{\circ}$ & $-5.8^{\circ}$ & $10.0^{\circ}$ & $8.3^{\circ}$ \\
F02 & $13.0^{\circ}$ & $10.8^{\circ}$ & $1.1^{\circ}$ & $1.5^{\circ}$ & $22.5^{\circ}$ & $22.0^{\circ}$ & $-3.3^{\circ}$ & $-4.6^{\circ}$ \\
F03 & $19.7^{\circ}$ & $17.0^{\circ}$ & $4.1^{\circ}$ & $6.2^{\circ}$ & $7.0^{\circ}$ & $7.6^{\circ}$ & $-0.5^{\circ}$ & $-0.1^{\circ}$ \\
F04 & $21.5^{\circ}$ & $18.1^{\circ}$ & $-8.3^{\circ}$ & $-8.8^{\circ}$ & $28.9^{\circ}$ & $26.9^{\circ}$ & $-6.8^{\circ}$ & $-7.2^{\circ}$ \\
M05 & $22.4^{\circ}$ & $15.2^{\circ}$ & $1.4^{\circ}$ & $3.7^{\circ}$ & $25.8^{\circ}$ & $25.8^{\circ}$ & $-3.2^{\circ}$ & $-4.4^{\circ}$ \\
M06 & $41.0^{\circ}$ & $37.3^{\circ}$ & $-15.4^{\circ}$ & $-13.7^{\circ}$ & $-9.1^{\circ}$ & $-2.1^{\circ}$ & $7.1^{\circ}$ & $6.4^{\circ}$ \\
M07 & $4.3^{\circ}$ & $2.5^{\circ}$ & $-1.7^{\circ}$ & $0.5^{\circ}$ & $4.2^{\circ}$ & $0.5^{\circ}$ & $-6.0^{\circ}$ & $-5.3^{\circ}$ \\
M08 & $25.5^{\circ}$ & $23.6^{\circ}$ & $-5.2^{\circ}$ & $-5.2^{\circ}$ & $-1.1^{\circ}$ & $-2.0^{\circ}$ & $14.0^{\circ}$ & $13.8^{\circ}$ \\
\hline
\end{tabular}

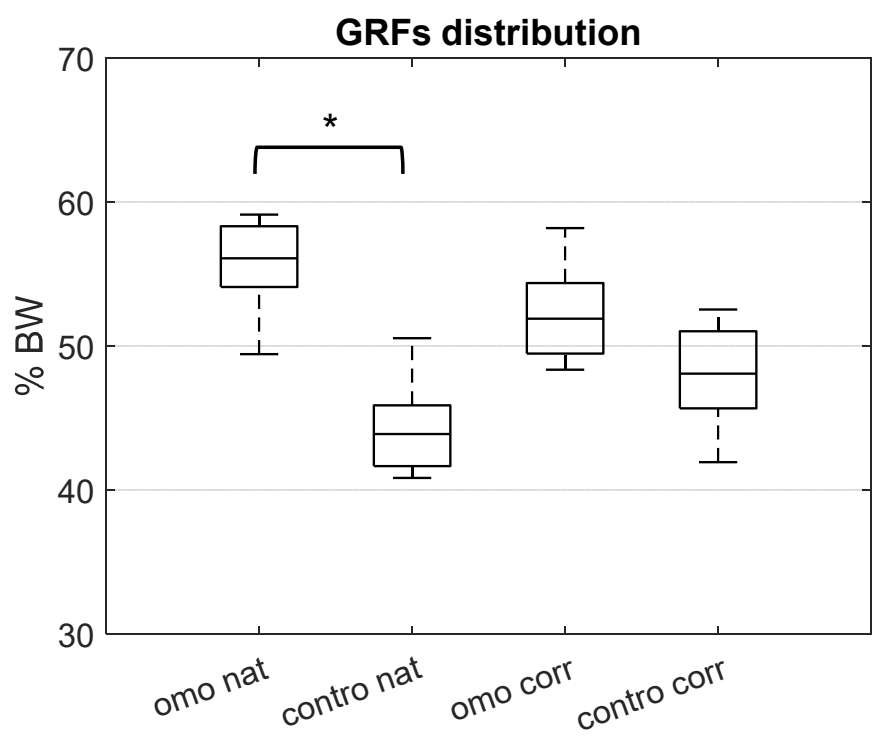

Figure 2. GRFs value separating omo and contralateral side, both in natural and correct standing posture. Significant $p$-value between columns is reported with asterisk. 
Figure 3 represents the scatter plot differences values of trunk sup-trunk inf angles (sagittal-blue, frontal-yellow) and omolateral ground reaction forces between natural and correct conditions, stressing the correlation between kinematics and body weight distribution. $R$ values ( 0.6 for the sagittal plane, -0.4 for the frontal plane) are depicted on the graph.

\section{Correlation}

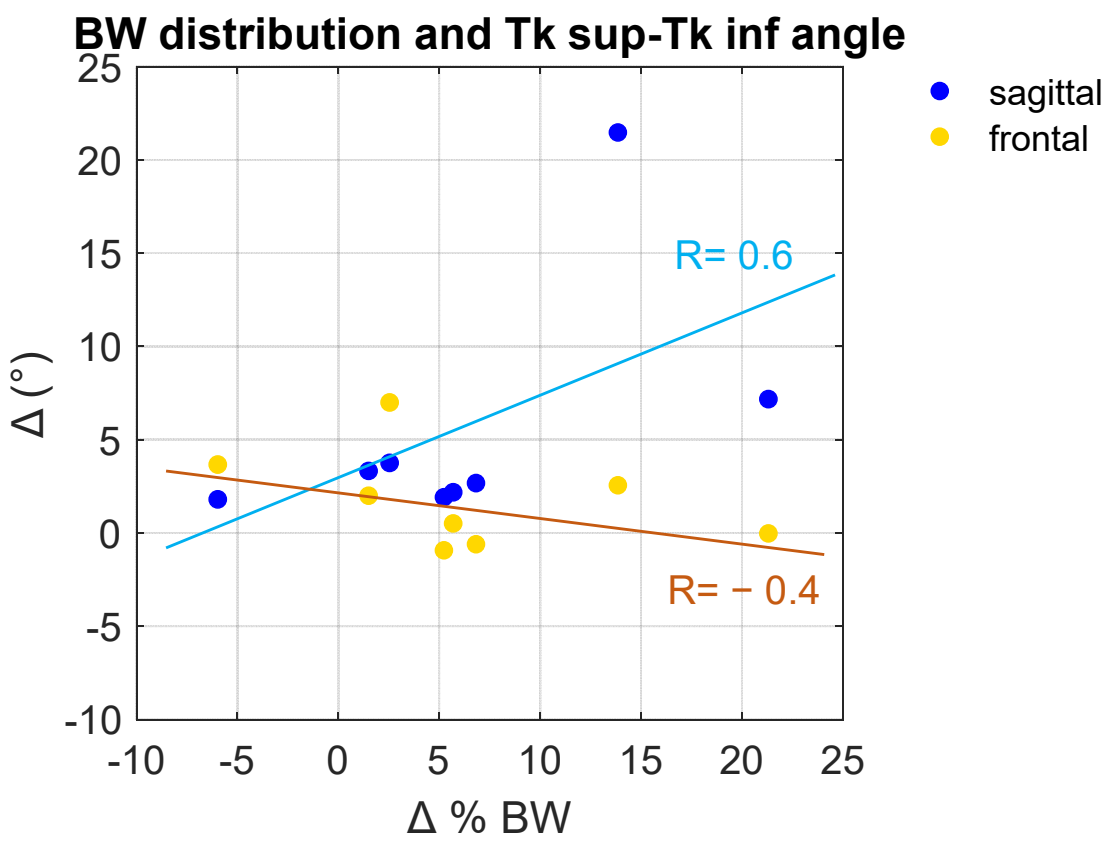

Figure 3. Correlation between the trunk superior-trunk inferior relative angles in sagittal (blue) and frontal (yellow) plane and GRFs in the omolateral side.

\subsection{Gait Trails}

Table 3 shows the mean and SD values of spatio-temporal parameters calculated inter-subject. The absolute difference between omolateral and contralateral sides was estimated for each subject and averaged inter-subject. Absolute values of mean differences are reported in Table $3(|\Delta|)$.

Table 3. Mean and SD value of spatio-temporal parameters during gait analysis, with the separation between omolateral and contralateral sides and the calculation of their differences.

\begin{tabular}{cccc}
\hline & $\begin{array}{c}\text { Spatio-Temporal Parameters } \\
\text { Mean (SD) }\end{array}$ \\
\hline Walking Speed (m/s) & Omolateral & $0.71(0.22)$ & Controlateral \\
\hline Stride Time (s) & $1.19(0.17)$ & $1.19(0.17)$ & I I \\
Step Time (s) & $0.59(0.08)$ & $0.60(0.10)$ & 0.02 \\
Stance Time (s) & $0.76(0.14)$ & $0.76(0.12)$ & 0.05 \\
Swing Time (s) & $0.42(0.09)$ & $0.42(0.09)$ & 0.03 \\
Stride Length (m) & $0.83(0.26)$ & $0.83(0.27)$ & 0.03 \\
Step Length (m) & $0.40(0.13)$ & $0.43(0.14)$ & 0.01 \\
Step Width (m) & $0.08(0.03)$ & $0.09(0.03)$ & 0.04 \\
Limp Index & $0.99(0.04)$ & $1.00(0.04)$ & 0.07 \\
Stance Duration (\%GC) & $64.00(5.49)$ & $64.42(4.22)$ & 2.36 \\
Swing Duration (\%GC) & $35.23(5.75)$ & $35.31(5.05)$ & 2.44 \\
\hline
\end{tabular}

Figures 4 and 5 depict an example of omolateral (dark green) and contralateral (red) lower limbs angular kinematics (hip, knee, ankle) of one PS patient in all three planes. Mean curves are represented with solid lines, SD values with dotted lines. In the same 
figures, the solid and dotted black lines represent the mean curve and the range of values of physiological gait kinematics, respectively [39].

Hip
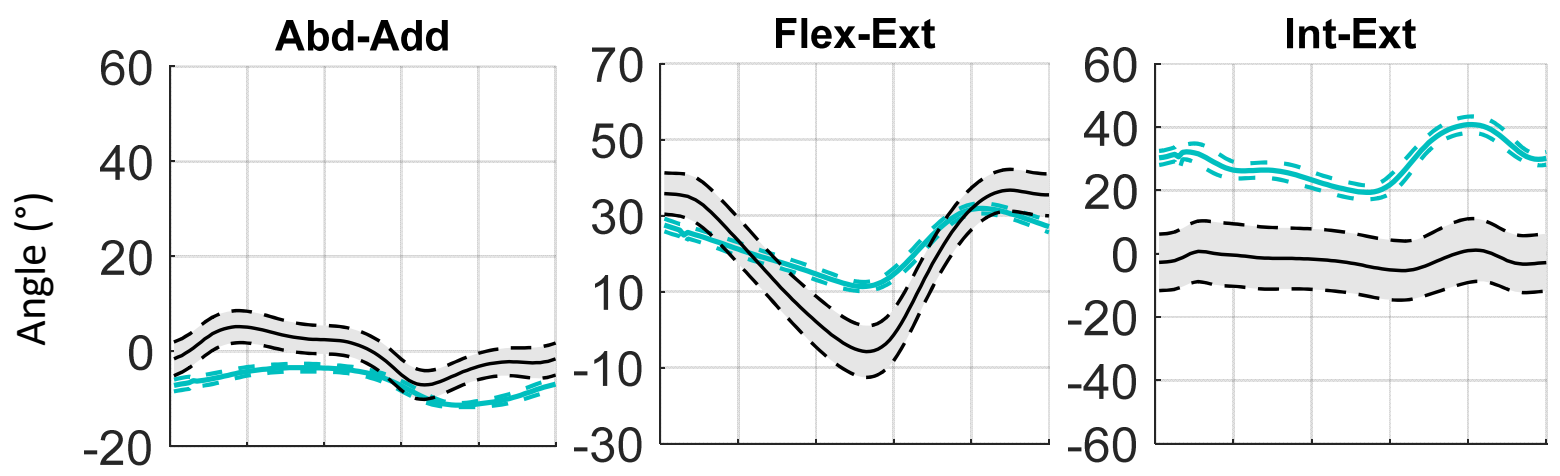

Knee

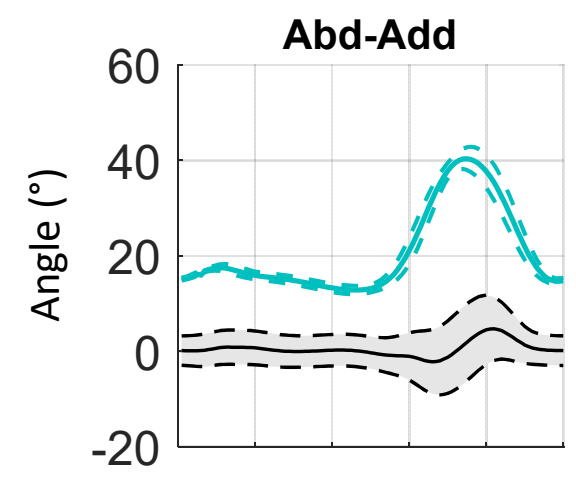

Flex-Ext
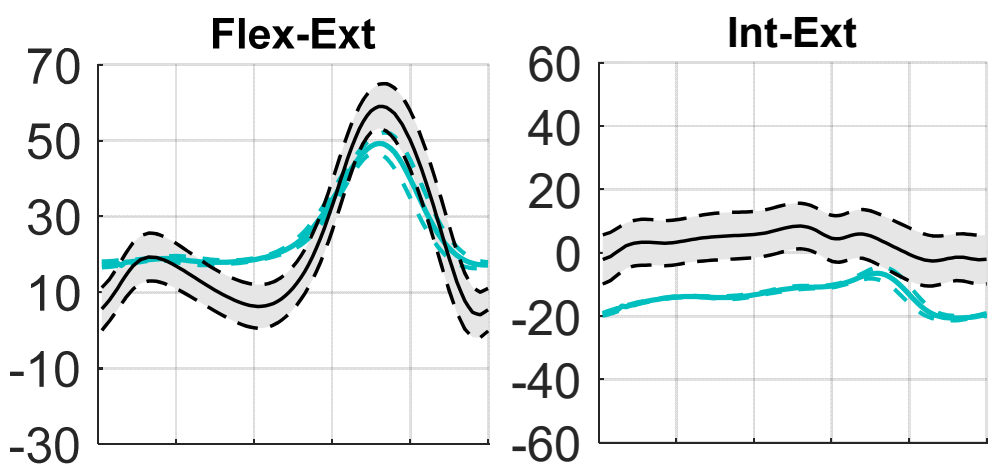

Ankle
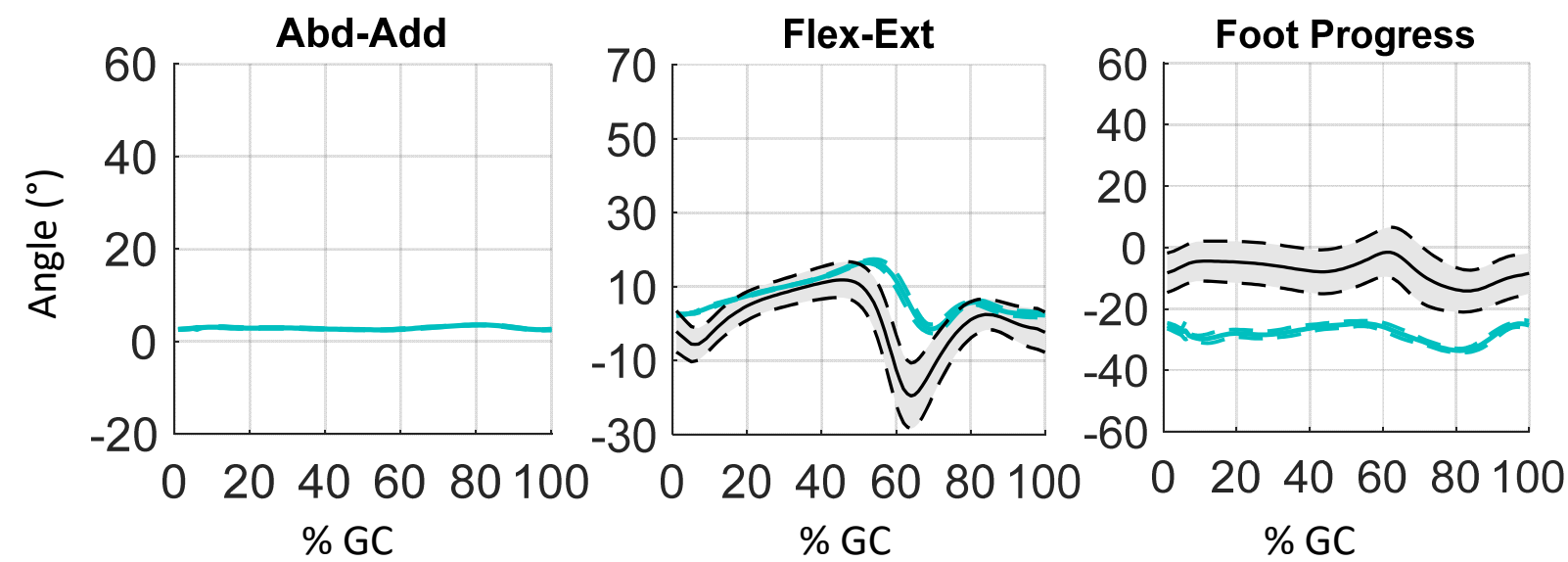

Figure 4. Example of 3D kinematics (Abduction-Adduction, Flexion-Extension, Internal-External rotation) of the lower body omolateral side of a PS patient during gait cycle $(\% \mathrm{GC})$. 

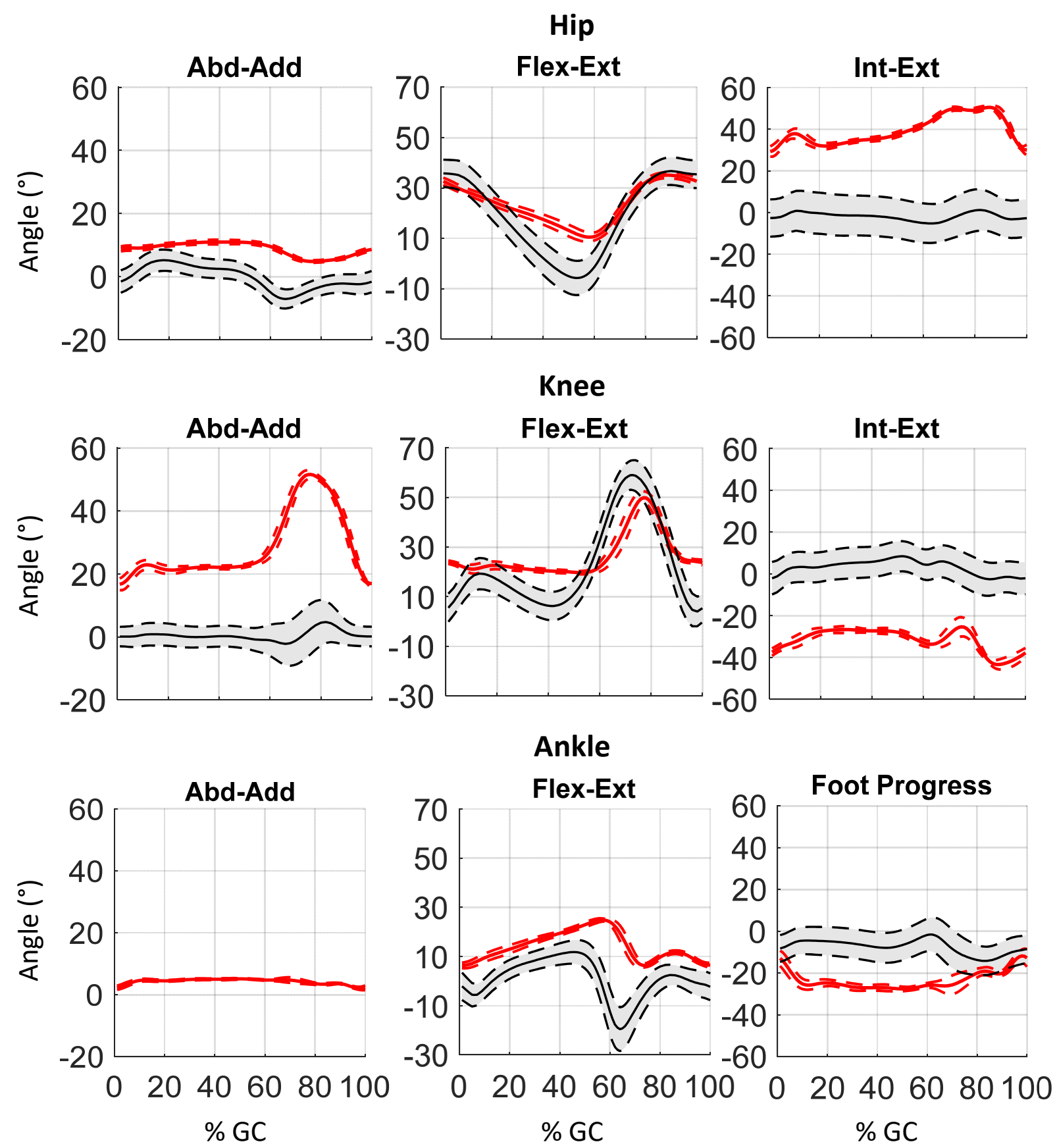

Figure 5. Example of 3D kinematics (Abduction-Adduction, Flexion-Extension, Internal-External rotation) of the lower body controlateral side during the gait cycle (\%GC) of a PS patient.

The mean and SD values of hip, knee and ankle ROMs calculated inter-subjects are reported in Table 4, with correspondent physiological ROMs of joints obtained in normal gait [39]. With the main attempt to underline any differences between left and right, omolateral and contralateral sides are maintained separately. 
Table 4. Mean and SD values of ROM in the three planes of lower body, separating omolateral and contralateral sides.

\begin{tabular}{|c|c|c|c|c|c|c|}
\hline \multicolumn{7}{|c|}{$\begin{array}{c}\text { ROM }\left({ }^{\circ}\right) \\
\text { Mean (SD) }\end{array}$} \\
\hline & \multicolumn{2}{|c|}{ Hip } & \multicolumn{2}{|c|}{ Knee } & \multicolumn{2}{|c|}{ Ankle } \\
\hline & Omo & Contro & Omo & Contro & Omo & Contro \\
\hline $\begin{array}{c}\text { Frontal } \\
\text { plane }\end{array}$ & $\begin{array}{c}9.22 \\
(2.58)\end{array}$ & $\begin{array}{l}10.17 \\
(3.87)\end{array}$ & $\begin{array}{l}17.80 \\
(7.81)\end{array}$ & $\begin{array}{c}20.41 \\
(13.21)\end{array}$ & $\begin{array}{c}2.19 \\
(1.74)\end{array}$ & $\begin{array}{c}3.55 \\
(1.68)\end{array}$ \\
\hline Physiological & \multicolumn{2}{|c|}{12.30} & \multicolumn{2}{|c|}{6.98} & \multicolumn{2}{|c|}{-} \\
\hline $\begin{array}{l}\text { Sagittal } \\
\text { plane }\end{array}$ & $\begin{array}{l}28.69 \\
(7.75)\end{array}$ & $\begin{array}{l}29.74 \\
(9.62)\end{array}$ & $\begin{array}{c}44.61 \\
(12.63)\end{array}$ & $\begin{array}{c}42.62 \\
(10.77)\end{array}$ & $\begin{array}{l}24.93 \\
(7.19)\end{array}$ & $\begin{array}{c}27.40 \\
(11.16)\end{array}$ \\
\hline Physiological & \multicolumn{2}{|c|}{42.48} & \multicolumn{2}{|c|}{55.11} & \multicolumn{2}{|c|}{31.63} \\
\hline $\begin{array}{l}\text { Transverse } \\
\text { plane }\end{array}$ & $\begin{array}{l}21.54 \\
(6.60)\end{array}$ & $\begin{array}{l}29.54 \\
(9.03)\end{array}$ & $\begin{array}{l}17.16 \\
(3.57)\end{array}$ & $\begin{array}{l}21.69 \\
(7.04)\end{array}$ & $\begin{array}{c}19.10 \\
(11.14)\end{array}$ & $\begin{array}{l}23.54 \\
(6.94)\end{array}$ \\
\hline Physiological & \multicolumn{2}{|c|}{6.51} & \multicolumn{2}{|c|}{11.04} & \multicolumn{2}{|c|}{12.64} \\
\hline
\end{tabular}

Figure 6 depicts an example of relative spine kinematics in one PS subject in frontal and sagittal planes obtained with the custom spinal multi-segment model. Relative angles between distal and proximal segments are reported. Mean curves are represented with solid lines, SD values with dotted lines. Kinematic curves are represented as a percentage of the gait cycle (\%GC).
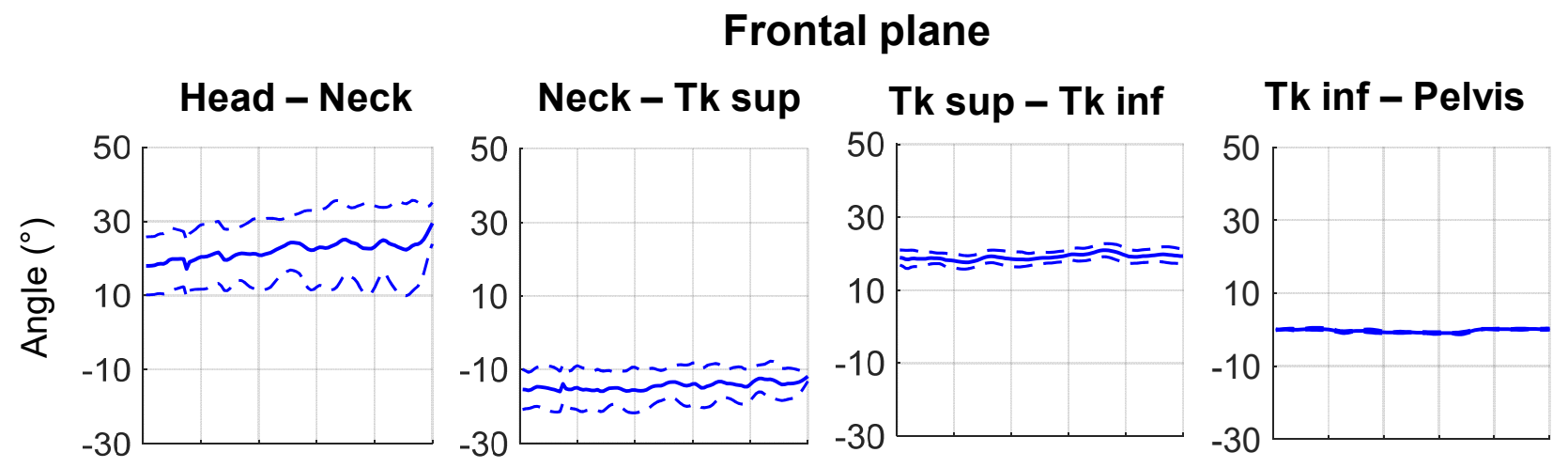

\section{Sagittal plane}

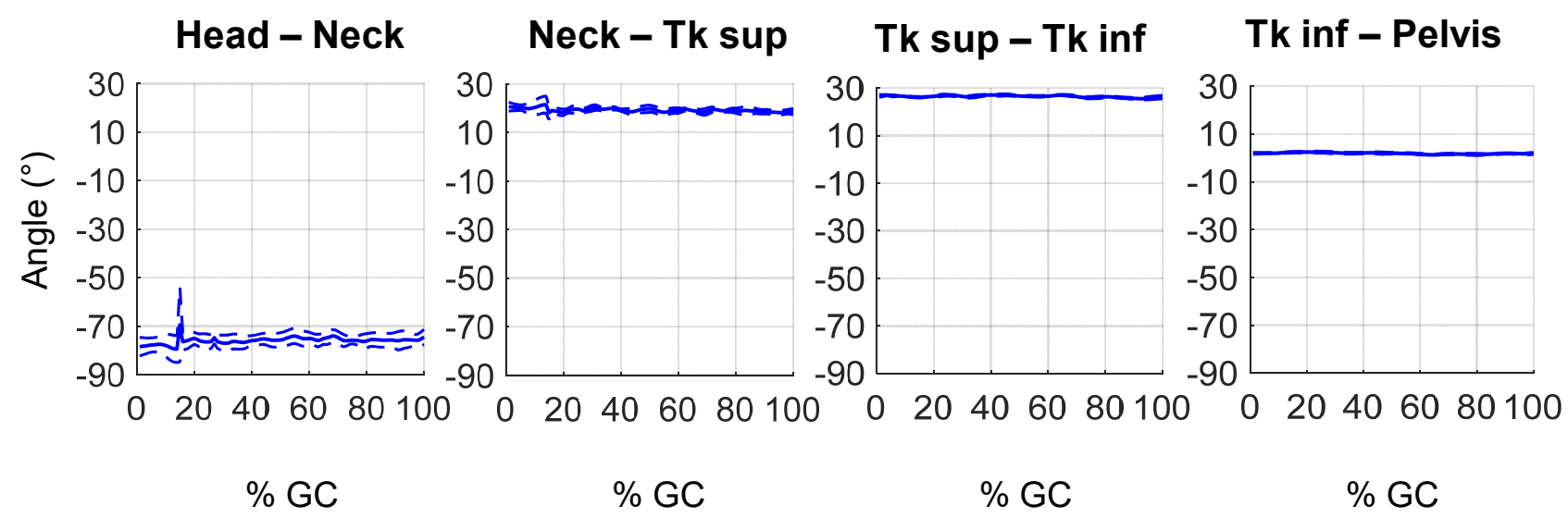

Figure 6. Example of 3D kinematics of the spine (distal segment relative to proximal segment) of a PS patient during gait cycle (\%GC). 


\section{Discussion}

The aim of the present study deals with the description and assessment of biomechanical analysis for the objective evaluation of movements disorders in patients with Pisa syndrome. The characteristic lateral deviation of the trunk in the frontal plane can also be correlated to other trunk deviations in the sagittal and transverse planes and causes asymmetrical posture, pain, difficulties in human daily activities, and unbalance. For all these reasons, a standardized procedure for the evaluation of motion alterations could be fundamental to quantify the impairments, to characterize the alterations of physiological patterns, to analyze relation with clinical variables and to verify the efficacy of treatments.

In the current study, two different common and functional daily activities were considered: static posture and gait.

\subsection{Static Posture}

Spine relative kinematics and load distribution were considered as parameters of interest during the static posture. Moreover, this task was performed by the subjects in two different conditions: natural and self-corrected posture, with the main attempt to evaluate their ability of correction and proprioception of asymmetry. All subjects showed an important flexion in the sagittal plane of the trunk superior segment with respect to the inferior one, with a strong variability among patients (range values between $4.3^{\circ}$ and $41.0^{\circ}$, Table 2). Nevertheless, all subjects revealed a significant ability to reduce this angle in case of self-correction (maximum of correction of $11.4^{\circ}$ for subject M01). On the contrary, the trunk inferior registered small flexion-extension angles in relation to the pelvis (range values between $15^{\circ}$ of extension and $4.1^{\circ}$ of flexion), with negligible differences in case of a correction. These differences can be expected, due to the point of separation between superior and inferior segments positioned in the vertebral range T12-L4. The angles in the frontal planes represent the lateral deviation of the trunk. Based on results, six patients revealed the greater contribution of lateral deviation at the superior segments, while two patients at the inferior ones. The inter-subject comparison highlights a large variability of lateral deviation, with absolute values in the range of $7.0^{\circ}$ and $28.9^{\circ}$. Kinematics of correct posture highlighted good proprioception of their wrong posture, as the complexity of compensation.

Considering the forces exchanged by the feet with the floor it is possible to analyze the load distribution on the lower limbs. Values were related to the total weight of patients and descriptive statistics are pointed out in Figure 2. The natural posture shows a significant asymmetry ( $p$-value $=0.02$ ) between the two sides, with greater load distribution on the omolateral foot (green bar). In the case of self-correction, all patients demonstrated a reduction of load on the omolateral side and a reduction of asymmetry and the difference between the omolateral and contralateral sides becomes statistically non-significant $(p$-value $=0.70)$. Comparing the natural and self-corrected conditions, results pointed out an asymmetrical distribution, but without stressing any significant difference. The results suggest an important difference between the two conditions, but additional investigations including a higher number of patients may be fundamental to confirm this aspect and to verify any statistical difference.

Kinematics and forces were related to quantifying the effects of the sagittal and frontal angle variations on the different loads' distribution. Figure 3 depicts a discrete correlation $(R=0.6)$ between the variation of trunk superior angle with respect to the trunk inferior segment on the sagittal plane and the variation of loads on the omolateral side, while a low correlation $(\mathrm{R}=-0.4)$ between the variation of angles on the frontal plane and loads' distribution. The negative sign of correlation means the relationship that exists between two variables is opposite all the time. Different strategies of correction could be implemented by subjects. Indeed, patients are expected to correct their posture with a greater muscular activation at the spinal level, or with a greater push from the contralateral foot. The adopted strategy could be verified considering the EMG activities of paraspinal muscles and with a larger population. One other important aspect that must be considered 
is the level of lateral deviation. Indeed, as already stressed, two patients showed greater deviation at inferior segment instead of superior ones. A deeper investigation considering all the spinal segments and a larger sample size might reveal additional considerations.

\subsection{Gait Trials}

Spatio-temporal parameters calculated during gait and reported in Table 3 are similar to results obtained in previous studies [24,32]. An important reduction of walking speed is one of the most incident effects $(0.71 \mathrm{~m} / \mathrm{s}$ instead of $1.10-1.30 \mathrm{~m} / \mathrm{s}$ registered in normal gait in the corresponding range of age $[40,41])$, while the high value of SD $(0.22 \mathrm{~m} / \mathrm{s})$ depicts an important variability among subjects. Differential parameters with the separation of the omolateral and contralateral sides are pointed out. Compared to physiological parameters and previous studies $[1,24,32]$, results highlighted a reduction of stride length $(0.83 \mathrm{~m})$ and, as a consequence, a reduction of step length, with an asymmetry between the omolateral $(0.40 \mathrm{~m})$ and contralateral $(0.43 \mathrm{~m})$ sides. Values of stance and swing duration expressed as a percentage of GC can be compared with expected values from normal gait, with a stance duration of $60 \%$ of gait and a swing duration of $40 \%$ of gait. PS patients reveal an increased stance duration $(65 \%)$ and a consequent reduction of swing $(35 \%)$. These values are similar to the results of PS patients' gait obtained by Geroin and colleagues in 2015 [24] and by Tramonti and colleagues in 2017 [32]. Due to the variability among subjects, absolute differences between omo and contralateral sides are evaluated for each subject, then averaged inter-subjects. Indeed, considering the mean values of variables, any asymmetries could be hidden. Absolute differences stress the asymmetry between the two sides for all spatio-temporal parameters (Table 3).

Figures 4 and 5 depict an example of lower body 3D kinematics of one PS patient. Despite the similar trend of the omolateral and contralateral kinematic curves, important alteration of gait patterns can be underlined. Indeed, considering the sagittal plane, the subject shows an important reduction of hip extension during the stance phase, pointing out a hip flexed pattern for all the gait cycles. This result is related to the high degree of trunk flexion shown by the patient and depicted in Figure 6. The knee joints show a flexed position during the stance phase and a reduction of knee flexion peak during the swing phase, both at the omo and contralateral sides. This alteration could be a compensative strategy for the reduction of unbalance caused by the trunk position. Finally, the ankle joints revealed an important reduction of plantarflexion during the pre-swing phases that reduces the contribution of limb push-off. Some important deviations could be highlighted considering frontal and transverse planes, as the increased internal rotation of the hip, the varus pattern of the knee and the external rotation of foot progress.

Comparing joint ROMs (Table 4) to physiological ones, in the sagittal planes all joints point out a significant reduction of ROMs. These reductions were previously obtained and discussed by Tramonti [32] and related to the reduction of stride length was pointed out. Nevertheless, it is also important to highlight the increased range of motions on frontal and transverse planes obtained for all joints. These increases might be identified as possible compensation for the wrong trunk posture assumed by the patients. Finally, asymmetries between the omo and contralateral sides can be stressed for all joints and in all planes. In particular, the omolateral side reveals a greater reduction of ROMs compared to the contralateral ones in the sagittal plane, while the contralateral sides revealed a greater increase in ROMs in the transverse and frontal planes.

As for the lower limbs, Figure 6 reports an example of trunk kinematics during gait for one patient. The segment that reveals greater variability during the gait cycle is the head, both in the sagittal and frontal planes. Considering the frontal plane, segments present a different side of lateral deviation. The trunk superior segment shows the lateral deviation due to the pathology, while the positions of the cervical segment and the head are probably compensation. The trunk inferior segment, in this case, reveals to be aligned with the pelvis one. Along the sagittal plane, important flexion of spinal segments is registered both at trunk superior and cervical segments. Nevertheless, a compensative hyper-extension of the 
head is performed by the subject to obtain a correct visual position during the gait motion. As in the frontal plane, also in this case the trunk inferior segment shows negligible rotation with respect to the pelvis segment.

\section{Conclusions}

In conclusion, the present study confirms the importance of a standardized biomechanical investigation of patients affected by Pisa syndrome. The analysis of standing posture and gait highlighted significant asymmetries in weight distributions, with a greater percentage of weight on the omolateral side on trunk deviation. Sagittal and frontal kinematics of spinal segments revealed important flexion and lateral flexion degrees among patients. In some cases, patients were able to reduce both forces and kinematics alterations with a proper request of corrections. During gait, spatio-temporal parameters and lower body kinematics depicted altered values and patterns compared to physiological ones. Asymmetries between omo- and contralateral sides could be stressed, as an important contribution of trunk deviation at different spinal levels, both in the sagittal and frontal planes.

Some limitations of the research can be pointed out. The most important being the small sample of participants, and the small number of motion repetitions due to difficulties for some patients in performing numerous sessions of different movements without fatigue. Moreover, the heterogeneity among subjects could cause a large variability in results. Patients were evaluated one time, without considering the possible positive effects of rehabilitation and clinical treatments (such as a botulinum toxin injection). A comparison of biomechanical parameters before and after treatments could highlight important results and could allow additional and deeper statistical analyses.

Future perspectives will consider a larger population and additional biomechanical parameters, such as surface EMG of paravertebral muscles and center of mass position. Balance tasks might be added to the protocol, with the main attempt of investigating proprioception and risk of falls for PS patients. The analysis of surface EMG at lumbar and thoracic levels with the separation of omo- and contralateral sides might reveal important considerations on the different muscular activation. The segmentation of the spine could underline the different contributions of each vertebra level to the movement. Moreover, objective parameters from motion capture systems could be correlated with subjective outcomes and clinical evaluations.

Author Contributions: Conceptualization, U.D. and C.A.A.; methodology, E.P., U.D. and C.A.A.; software, E.P.; validation and data analysis, E.P. and L.G.; formal analysis, E.P.; resources, U.D. and C.A.A.; writing—original draft preparation, E.P.; writing—review and editing, E.P., L.G. and C.A.A.; supervision, L.G.; project administration, U.D. All authors have read and agreed to the published version of the manuscript.

Funding: This research received no external funding.

Institutional Review Board Statement: The study was conducted according to the guidelines of the Declaration of Helsinki, and approved by the Ethics Committee of "Comitato Etico Interaziendale, A.O.U. Città della Salute e della Scienza di Torino-A.O. Ordine Mauriziano di Torino-A.S.L. Città di Torino" (Turin-Italy, protocol code 0045381, date of approval 30 April 2018).

Informed Consent Statement: Informed consent was obtained from all subjects involved in the study. Data Availability Statement: Not applicable.

Acknowledgments: Authors acknowledge the Clinical Unit staff of the Unità Spinale Unipolare di Torino and subjects that participated in the experiments.

Conflicts of Interest: The authors declare no conflict of interest. 


\section{References}

1. Digo, E.; Agostini, V.; Pastorelli, S.; Gastaldi, L.; Panero, E. Gait Phases Detection in Elderly using Trunk-MIMU System. In Proceedings of the 14th International Joint Conference on Biomedical Engineering Systems and Technologies, Vienna, Austria, 11-13 February 2021; Scitepress-Science and Technology Publications: Southampton, UK, 2021; pp. 58-65.

2. Morris, M.E.; Huxham, F.; McGinley, J.; Dodd, K.; Iansek, R. The biomechanics and motor control of gait in Parkinson disease. Clin. Biomech. 2001, 16, 459-470. [CrossRef]

3. Debû, B.; Godeiro, C.D.O.; Lino, J.C.; Moro, E. Managing Gait, Balance, and Posture in Parkinson's Disease. Curr. Neurol. Neurosci. Rep. 2018, 18, 23. [CrossRef] [PubMed]

4. Doherty, K.M.; van de Warrenburg, B.P.; Peralta, M.C.; Moriyama, L.S.; Azulay, J.-P.; Gershanik, O.S.; Bloem, B.R. Postural deformities in Parkinson's disease. Lancet Neurol. 2011, 10, 538-549. [CrossRef]

5. Di Matteo, A.; Fasano, A.; Squintani, G.; Ricciardi, L.; Bovi, T.; Fiaschi, A.; Barone, P.; Tinazzi, M. Lateral trunk flexion in Parkinson's disease: EMG features disclose two different underlying pathophysiological mechanisms. J. Neurol. 2010, 258, 740-745. [CrossRef] [PubMed]

6. Bonanni, L.; Thomas, A.M.; Varanese, S.; Scorrano, V.; Onofrj, M. Botulinum toxin treatment of lateral axial dystonia in parkinsonism. Mov. Disord. 2007, 22, 2097-2103. [CrossRef]

7. Tinazzi, M.; Geroin, C.; Gandolfi, M.; Smania, N.; Tamburin, S.; Morgante, F.; Fasano, A. Pisa syndrome in Parkinson's disease: An integrated approach from pathophysiology to management. Mov. Disord. 2016, 31, 1785-1795. [CrossRef]

8. Castrioto, A.; Piscicelli, C.; Pérennou, D.; Krack, P.; Debû, B. The pathogenesis of Pisa syndrome in Parkinson's disease. Mov. Disord. 2014, 29, 1100-1107. [CrossRef]

9. Doherty, K.M.; Davagnanam, I.; Molloy, S.; Moriyama, L.S.; Lees, A.J. Pisa syndrome in Parkinson's disease: A mobile or fixed deformity? J. Neurol. Neurosurg. Psychiatry 2013, 84, 1400-1403. [CrossRef] [PubMed]

10. Brognara, L.; Palumbo, P.; Grimm, B.; Palmerini, L. Assessing Gait in Parkinson's Disease Using Wearable Motion Sensors: A Systematic Review. Diseases 2019, 7, 18. [CrossRef] [PubMed]

11. Jarchi, D.; Pope, J.; Lee, T.K.M.; Tamjidi, L.; Mirzaei, A.; Sanei, S. A Review on Accelerometry-Based Gait Analysis and Emerging Clinical Applications. IEEE Rev. Biomed. Eng. 2018, 11, 177-194. [CrossRef]

12. Celik, Y.; Stuart, S.; Woo, W.; Godfrey, A. Gait analysis in neurological populations: Progression in the use of wearables. Med. Eng. Phys. 2021, 87, 9-29. [CrossRef] [PubMed]

13. Pau, M.; Leban, B.; Deidda, M.; Putzolu, F.; Porta, M.; Coghe, G.; Cocco, E. Kinematic Analysis of Lower Limb Joint Asymmetry During Gait in People with Multiple Sclerosis. Symmetry 2021, 13, 598. [CrossRef]

14. Higgs, J.; Diamond, L.; Saxby, D.; Constantinou, M.; Barrett, R. Individuals with Unilateral Mild-to-Moderate Hip Osteoarthritis Exhibit Lower Limb Kinematic Asymmetry during Walking But Not Sit-to-Stand. Symmetry 2021, 13, 768. [CrossRef]

15. Kim, W.-S.; Choi, H.; Jung, J.-W.; Yoon, J.S.; Jeoung, J.H. Asymmetry and Variability Should Be Included in the Assessment of Gait Function in Poststroke Hemiplegia With Independent Ambulation During Early Rehabilitation. Arch. Phys. Med. Rehabil. 2021, 102, 611-618. [CrossRef]

16. Carollo, J.; De, S.; Akuthota, V. Evidence-Based Physiatry: Clinical Decision-Making with Instrumented Gait Analysis. Am. J. Phys. Med. Rehabil. 2020, 99, 265-266. [CrossRef]

17. Zanardi, A.P.J.; Martinez, F.G.; da Silva, E.S.; Casal, M.Z.; Martins, V.F. Effects of nordic walking on gait symmetry in mild Parkinson's disease. Symmetry 2019, 11, 1481. [CrossRef]

18. Di Biase, L.; Di Santo, A.; Caminiti, M.L.; De Liso, A.; Shah, S.A.; Ricci, L.; Di Lazzaro, V. Gait Analysis in Parkinson's Disease: An Overview of the Most Accurate Markers for Diagnosis and Symptoms Monitoring. Sensors 2020, 20, 3529. [CrossRef]

19. Nardello, F.; Bertoli, E.; Bombieri, F.; Bertucco, M.; Monte, A. The Effect of a Secondary Task on Kinematics during Turning in Parkinson's Disease with Mild to Moderate Impairment. Symmetry 2020, 12, 1284. [CrossRef]

20. Ye, X.; Lou, D.; Ding, X.; Xie, C.; Gao, J.; Lou, Y.; Cen, Z.; Xiao, Y.; Miao, Q.; Xie, F.; et al. A clinical study of the coronal plane deformity in Parkinson disease. Eur. Spine J. 2017, 26, 1862-1870. [CrossRef]

21. Huh, Y.E.; Kim, K.; Chung, W.-H.; Youn, J.; Kim, S.; Cho, J.W. Pisa Syndrome in Parkinson's Disease: Pathogenic Roles of Verticality Perception Deficits. Sci. Rep. 2018, 8, 1804. [CrossRef]

22. Todisco, M.; Pozzi, N.G.; Zangaglia, R.; Minafra, B.; Servello, D.; Ceravolo, R.; Alfonsi, E.; Fasano, A.; Pacchetti, C. Pisa syndrome in Idiopathic Normal Pressure Hydrocephalus. Park. Relat. Disord. 2019, 66, 40-44. [CrossRef]

23. Tinazzi, M.; Juergenson, I.; Squintani, G.; Vattemi, G.; Montemezzi, S.; Censi, D.; Barone, P.; Bovi, T.; Fasano, A. Pisa syndrome in Parkinson's disease: An electrophysiological and imaging study. J. Neurol. 2013, 260, 2138-2148. [CrossRef]

24. Geroin, C.; Smania, N.; Schena, F.; Dimitrova, E.; Verzini, E.; Bombieri, F.; Nardello, F.; Tinazzi, M.; Gandolfi, M. Does the Pisa syndrome affect postural control, balance, and gait in patients with Parkinson's disease? An observational cross-sectional study. Park. Relat. Disord. 2015, 21, 736-741. [CrossRef]

25. Tinazzi, M.; Gandolfi, M.; Artusi, C.A.; Lanzafame, R.; Zanolin, E.; Ceravolo, R.; Capecci, M.; Andrenelli, E.; Ceravolo, M.G.; Bonanni, L.; et al. Validity of the wall goniometer as a screening tool to detect postural abnormalities in Parkinson's disease. Park. Relat. Disord. 2019, 69, 159-165. [CrossRef] [PubMed]

26. Formaggio, E.; Masiero, S.; Volpe, D.; Demertzis, E.; Gallo, L.; Del Felice, A. Lack of inter-muscular coherence of axial muscles in Pisa syndrome. Neurol. Sci. 2019, 40, 1465-1468. [CrossRef] 
27. Margraf, N.G.; Rogalski, M.; Deuschl, G.; Kuhtz-Buschbeck, J.P. Trunk muscle activation pattern in parkinsonian camptocormia as revealed with surface electromyography. Park. Relat. Disord. 2017, 44, 44-50. [CrossRef] [PubMed]

28. Kataoka, H.; Sawa, N.; Ueno, S. Identification of a new target muscle for treatment in patients with Parkinson's disease who have lateral trunk flexion? J. Neurol. Sci. 2015, 358, 435-439. [CrossRef]

29. Frazzitta, G.; Balbi, P.; Gotti, F.; Maestri, R.; Sabetta, A.; Caremani, L.; Gobbi, L.; Capobianco, M.; Bera, R.; Giladi, N.; et al. Pisa Syndrome in Parkinson's Disease: Electromyographic Aspects and Implications for Rehabilitation. Park. Dis. 2015, 2015, 437190. [CrossRef]

30. Cano-De-La-Cuerda, R.; Vela-Desojo, L.; Moreno-Verdú, M.; Ferreira-Sánchez, M.D.R.; Macías-Macías, Y.; Miangolarra-Page, J.C. Trunk Range of Motion Is Related to Axial Rigidity, Functional Mobility and Quality of Life in Parkinson's Disease: An Exploratory Study. Sensors 2020, 20, 2482. [CrossRef] [PubMed]

31. Schlenstedt, C.; Boße, K.; Gavriliuc, O.; Wolke, R.; Granert, O.; Deuschl, G.; Margraf, N.G. Quantitative assessment of posture in healthy controls and patients with Parkinson's disease. Park. Relat. Disord. 2020, 76, 85-90. [CrossRef]

32. Tramonti, C.; Di Martino, S.; Unti, E.; Frosini, D.; Bonuccelli, U.; Rossi, B.; Ceravolo, R.; Chisari, C. Gait dynamics in Pisa syndrome and Camptocormia: The role of stride length and hip kinematics. Gait Posture 2017, 57, 130-135. [CrossRef]

33. Crawford, N.R.; Yamaguchi, G.T.; Dickman, C.A. A new technique for determining 3-D joint angles: The tilt/twist method. Clin. Biomech. 1999, 14, 153-165. [CrossRef]

34. Leardini, A.; Biagi, F.; Merlo, A.; Belvedere, C.; Benedetti, M.G. Multi-segment trunk kinematics during locomotion and elementary exercises. Clin. Biomech. 2011, 26, 562-571. [CrossRef]

35. Needham, R.; Naemi, R.; Healy, A.; Chockalingam, N. Multi-segment kinematic model to assess three-dimensional movement of the spine and back during gait. Prosthet. Orthot. Int. 2016, 40, 624-635. [CrossRef]

36. Crosbie, J.; Vachalathiti, R.; Smith, R. Patterns of spinal motion during walking. Gait Posture 1997, 5, 6-12. [CrossRef]

37. Panero, E.; Digo, E.; Ferrarese, V.; Dimanico, U.; Gastaldi, L. Multi-Segments Kinematic Model of the Human Spine during Gait. In Proceedings of the 2021 IEEE International Symposium on Medical Measurements and Applications (MeMeA), Lausanne, Switzerland, 23-25 June 2021.

38. Ratner, B. The correlation coefficient: Its values range between 1/1, or do they. J. Target. Meas. Anal. Mark. 2009, 17, 139-142. [CrossRef]

39. Vicon I Award Winning Motion Capture Systems. Available online: https://www.vicon.com/ (accessed on 1 July 2021).

40. Oberg, T.; Karsznia, A.; Oberg, K. Basic gait parameters: Reference data for normal subjects, 10-79 years of age. J. Rehabil. Res. Dev. 1993, 30, 210-223. [PubMed]

41. Bohannon, R.W.; Andrews, A.W. Normal walking speed: A descriptive meta-analysis. Physiotherapy 2011, 97, 182-189. [CrossRef] [PubMed] 